\title{
A multi-technique investigation of the nanoporosity of cement paste
}

\author{
Hamlin M. Jennings ${ }^{\mathrm{a}, \mathrm{b}, *}$, Jeffrey J. Thomas ${ }^{\mathrm{a}}$, Julia S. Gevrenov ${ }^{\mathrm{a}, \mathrm{b}}$, Georgios Constantinides ${ }^{\mathrm{c}}$, \\ Franz-Josef Ulm ${ }^{\mathrm{c}}$

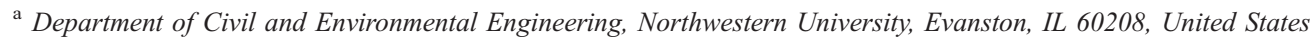 \\ ${ }^{\mathrm{b}}$ Department of Materials Science and Engineering, Northwestern University, Evanston, IL 60208, United States \\ ${ }^{c}$ Department of Civil and Environmental Engineering, Massachusetts Institute of Technology, Cambridge, MA 02139, United States
}

Received 16 December 2005; accepted 22 March 2006

\begin{abstract}
The nanometer-scale structure of cement paste, which is dominated by the colloidal-scale porosity within the C-S-H gel phase, has a controlling effect on concrete properties but is difficult to study due to its delicate structure and lack of long-range order. Here we present results from three experimental techniques that are particularly suited to analyzing disordered nanoporous materials: small-angle neutron scattering (SANS), weight and length changes during equilibrium drying, and nanoindentation. Particular attention is paid to differences between pastes of different ages and cured at different temperatures. The SANS and equilibrium drying results indicate that hydration of cement paste at $20^{\circ} \mathrm{C}$ forms a low-density (LD) C-S-H gel structure with a range of gel pore sizes and a relatively low packing fraction of solid particles. This fine structure may persist indefinitely under saturated conditions. However, if the paste is dried or is cured at elevated temperatures $\left(60^{\circ} \mathrm{C}\right.$ or greater) the structure collapses toward a denser (less porous) and more stable configuration with fewer large gel pores, resulting in a greater amount of capillary porosity. Nanoindentation measurements of pastes cured at different temperatures demonstrate in all cases the existence of two C-S-H structures with different characteristic values of the indentation modulus. The average value of the modulus of the LD C-S-H is the same for all pastes tested to date, and a micromechanical analysis indicates that this value corresponds to the denser and more stable configuration of LD C-S-H. The experimental results presented here are interpreted in terms of a previously proposed quantitative "colloid" model of C-S-H gel, resulting in an improved understanding of the microstructural changes associated with drying and heat curing.
\end{abstract}

(c) 2006 Elsevier Ltd. All rights reserved.

Keywords: Calcium-silicate-hydrate (C-S-H); Pore size distribution; Drying; Temperature; Elastic moduli

\section{Introduction}

The microstructures of cement-based materials are so complex that they are seldom evaluated quantitatively. Only the larger features such as the volume of capillary pores and the distribution of air voids have been correlated to properties such as strength and resistance to repeated freezing. Yet it is the smallest gel pores associated with the calcium-silicate-hydrate (C-S-H) phase that control properties such as creep and shrinkage. Recently, multi-scale modeling has proven to be a

\footnotetext{
* Corresponding author. Department of Civil and Environmental Engineering, Northwestern University, Evanston, IL 60208, United States. Tel.: +1 847491 5282; fax: +1 8474914011.

E-mail addresses: h-jennings@northwestern.edu (H.M. Jennings), jthomas@northwestern.edu (J.J. Thomas).
}

promising approach for correlating the nanometer-level structure of C-S-H to the bulk properties of cement paste and concrete [1]. However, for a number of reasons that include problems with resolving the delicate nanoscale structure and understanding the changes that occur with drying, age, temperature, mechanical loading, and other environmental variables, the structure of C-S-H at the nanometer scale has not been unambiguously characterized.

The goals of this paper are twofold. First, we present experimental results from three techniques that, particularly when interpreted together, give a more complete and detailed picture of the nanostructure of the C-S-H gel phase as a function of aging, drying, and heating. The results from these techniques, namely small-angle scattering, equilibrium drying, and nanoindentation, are discussed in Sections 3-5. The second goal of the paper is to extend a quantitative structural model of the C-S-H 
gel [2] to account for this new experimental data. This is the subject of Section 6.

\section{Experimental}

The specimens analyzed by SANS and by nanoindentation were made from commercial white portland cement (WPC), while the specimens analyzed by equilibrium drying were made from a commercial Type I portland cement (OPC). All pastes were mixed at a water/cement ratio of 0.5 by weight and cured under saturated conditions. The curing temperature was controlled at 20,60, or (for one specimen) $80{ }^{\circ} \mathrm{C}$ using programmable water baths. Specimens cured at $60^{\circ} \mathrm{C}$ for at least the first $24 \mathrm{~h}$ of hydration are considered "heat cured", whereas specimens heated to $60{ }^{\circ} \mathrm{C}$ after an initial period of curing at $20^{\circ} \mathrm{C}$ are considered "heat treated". Curing times varied and are given in the text. For the SANS measurements, thin $(\sim 0.6 \mathrm{~mm})$ coupons were sliced from a larger block of paste using a waterlubricated diamond saw. Specimens for nanoindentation were prepared in a similar manner, but were approximately $1 \mathrm{~cm}$ thick. In addition, nanoindentation specimens were ground and polished in six stages of decreasing fineness, beginning with silicon carbide paper and finishing with a $250 \mathrm{~nm}$ diamond particle suspension. Care was taken to keep the opposite faces of the specimens flat and parallel, as any deviation could influence the angle of indentation. As a final step, the specimens were cleaned in an ultrasonic bath containing a saturated $\mathrm{CaO}$ solution. For the equilibrium drying experiments, slender bars of OPC paste measuring $150 \times 5 \times 5 \mathrm{~mm}$ were cut from larger bars of paste and fitted with steel endpoints using waterproof epoxy.

The SANS measurements were conducted at the National Center for Neutron Research in Gaithersburg, MD, using a neutron wavelength of $0.8 \mathrm{~nm}$ and an effective range of scattering vector of $0.02-2.2 \mathrm{~nm}^{-1}$. More details on the experimental configuration and the data normalization are given in [3]. Nanoindentation measurements were conducted using a NanoTest 200 instrument in the Department of Materials Science and Engineering at MIT. More details are given in [4].

For the equilibrium drying experiments, initially saturated bars were progressively dried in stepwise fashion by placing them in sealed desiccators containing saturated salt solutions with different equilibrium relative humidities $(\mathrm{RH})$ ranging from $85 \%$ to $7 \%$. At regular intervals (typically $1 \mathrm{~d}$ ) the bars were weighed and the length was measured using a micrometer mounted on a customized stand. Only when the length and weight had stabilized at one RH was the specimen moved to the desiccator containing the next-lowest RH. In some cases the specimens were resaturated and then the entire drying cycle was repeated.

\section{Small-angle scattering measurements}

Small-angle neutron scattering (SANS) provides a great deal of information about the micro- and nanostructures of disordered heterogeneous materials. The most versatile and sensitive SANS instruments can produce a statistically averaged characterization across length scales ranging from approximately $1 \mathrm{~nm}$ to greater than $1 \mu \mathrm{m}$. SANS has some important advantages for characterizing the nanometer-level structure of cement-based materials when compared to other techniques such as gas sorption and TEM. The SANS analysis is non-invasive and does not require drying, and can even be conducted in situ without disturbing the hydration process.

When the scattered neutron intensity from a cementitious material is plotted against the scattering vector, $Q$, (proportional to the angle of scatter) three regimes of interest are generally observed (see Fig. 1). At highest $Q$ (representing the smallest microstructural features) the scattering follows the Porod law and gives a direct measurement of the total internal surface area, $S_{\mathrm{T}}$. At lower $Q$-values two power-law regimes are observed with different slopes, corresponding to volume fractal and surface fractal scaling behavior $[3,5,6]$. The volume fractal scattering is associated with the arrangement (packing) of primary C-S-H gel particles (of size $\sim 4-5 \mathrm{~nm}$ ) and is limited to relatively high $Q$ values. The surface fractal scaling can be associated with the deposition of hydration product onto the originally smooth surface of the cement grains. This fractal roughness extends upward in size to include relatively large (micron-scale) features such as calcium hydroxide crystals. As hydration proceeds, the volume fractal intensity increases while the surface fractal intensity tends to decrease as the original boundaries of the clinker grains become obscured. Under some conditions, such as in calcium chloride accelerated paste [7], the surface fractal regime can essentially disappear.

Fig. 1 shows SANS data for an $8 \mathrm{~d}$ old WPC paste cured at $20^{\circ} \mathrm{C}$, an $8 \mathrm{~d}$ old WPC paste cured at $60^{\circ} \mathrm{C}$ for the first $24 \mathrm{~h}$ only, and a $28 \mathrm{~d}$ old WPC paste cured at $80^{\circ} \mathrm{C}$. The scattering curves for all three pastes have the same general shape, suggesting that the fundamental microstructure is not radically changed by high

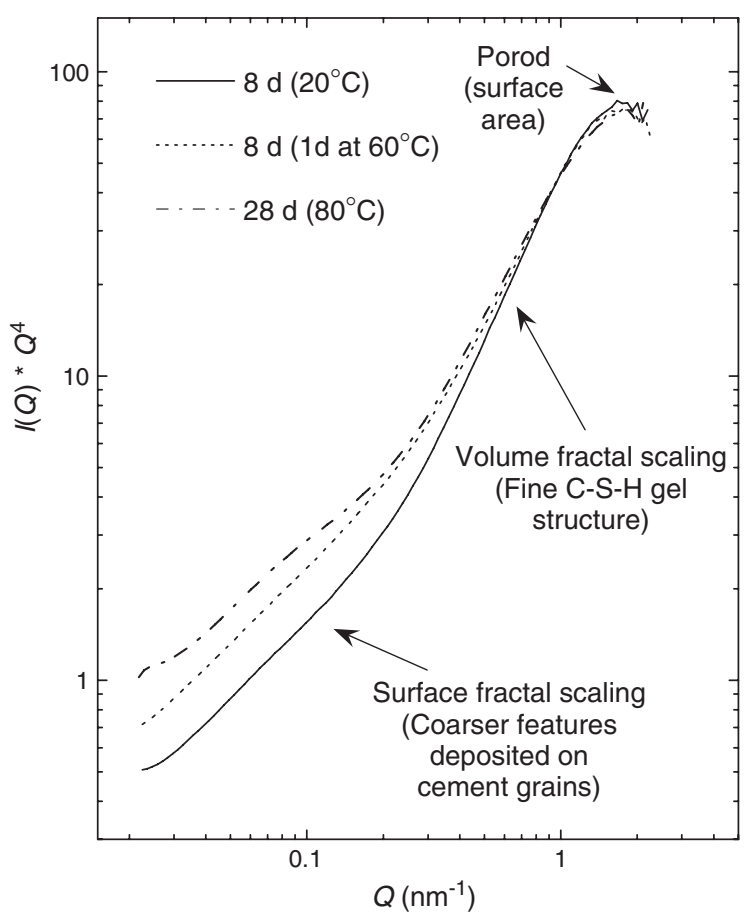

Fig. 1. SANS data for normal and heat cured WPC paste. 
temperature curing, but the surface fractal scattering is more prominent with heat curing. This is indicative of a more heterogeneous microstructure, where the original cement grains do not blend smoothly into the outer hydration product [8]. This is consistent with observations of denser hydration product $[9,10]$ and lower strength [11] in heat cured pastes.

To obtain a more quantitative characterization of the microstructure, SANS data from a wide range of $Q$ (such as that plotted in Fig. 1) can be fit with a model that includes appropriate terms for the volume and surface fractal scattering described above $[6,8]$. Here we focus on the volume fractal regime of the data (associated with the C-S-H gel), in which the scattered intensity varies as $Q^{-D \mathrm{~V}}$, where $D_{\mathrm{V}}$ is the volume fractal exponent. A volume fractal (also known as a mass fractal) is a two-phase region formed from particles of a characteristic size, $a_{\mathrm{S}}$, that are packed together in such a way that the structure exhibits scale invariance (i.e. fractal behavior). For cement paste these primary particles are modeled as C-S-H "globules" that have the measured characteristic size of $\sim 5 \mathrm{~nm}$ and, while not necessarily spherical, are roughly equiaxed. It should be noted that AFM studies [12] have reported that the primary C-S-H gel particles have a lamellar, or sheetlike shape, with a thickness of $5 \mathrm{~nm}$ but up to $60 \mathrm{~nm}$ in width, while TEM studies [13], on the other hand, have presented evidence for elongated or fibrous particles, also with a minimum dimension of a few nanometers. However, the SANS response from cement paste does not exhibit any of the hallmarks of elongated or sheetlike particles, unless the C-S-H phase is decalcified [3].

Inside a volume fractal, the volume fraction of solid contained within an imaginary sphere of radius $d$ centered on a fixed point decreases with increasing $d$, as long as $a_{\mathrm{S}},<d<\xi_{\mathrm{V}}$, where $\xi_{\mathrm{V}}$ is the correlation length. The volume fraction of solid material within an isolated volume fractal region can be described as:

$\eta_{\mathrm{VF}}=\eta\left(\frac{\xi_{\mathrm{V}}}{a_{\mathrm{S}}}\right)^{D_{\mathrm{V}}-3}$

where $\eta$, the local maximum packing fraction at the scale of the individual particles, is also determined from the fractal microstructure model. The C-S-H gel structure in cement paste consists of multiple volume fractal regions that grow into each other and overlap during hydration [5], such that the actual average packing density of the $\mathrm{C}-\mathrm{S}-\mathrm{H}$ is expected to be higher than that predicted by Eq. (1). However, the ratio $\eta_{\mathrm{VF}} / \eta$ provides a relative measure of the C-S-H packing density that is useful for direct comparisons between different specimens. A model fit to the data also provides the volume fraction, $\phi_{\mathrm{CSH}}$, of solid volume fractal material (i.e. particles of size $a_{\mathrm{S}}$ ) within the entire specimen volume penetrated by the neutron beam. This is essentially a measure of the amount of LD C-S-H (without gel pores) in the paste.

Table 1 lists the surface area and the volume fractal parameters described above for the data shown in Fig. 1, along with data for a mature WPC paste cured at $20^{\circ} \mathrm{C}$. From Table 1 it can be seen that when cement paste is hydrated at room temperature there is little change in the surface area, fundamental C-S-H gel particle size, or volume fractal exponent between the ages of
Table 1

Total surface area and volume fractal parameters determined from SANS measurements of WPC paste specimens mixed at a water/cement ratio of 0.5 and given the curing time and temperature indicated

\begin{tabular}{|c|c|c|c|c|}
\hline & \multirow{2}{*}{$\begin{array}{l}8 \mathrm{~d} \\
\left(20^{\circ} \mathrm{C}\right)\end{array}$} & \multirow{2}{*}{$\begin{array}{l}2.5 \mathrm{y} \\
\left(20^{\circ} \mathrm{C}\right)\end{array}$} & $1 \mathrm{~d}\left(60{ }^{\circ} \mathrm{C}\right)$ & \multirow{2}{*}{$\begin{array}{l}28 \mathrm{~d} \\
\left(80^{\circ} \mathrm{C}\right)\end{array}$} \\
\hline & & & $+7 \mathrm{~d}\left(20^{\circ} \mathrm{C}\right)$ & \\
\hline Surface area, $S_{\mathrm{T}}\left(\mathrm{m}^{2} / \mathrm{cm}^{3}\right)$ & $128(6)$ & $135(7)$ & $127(6)$ & $108(5)$ \\
\hline$(\mathrm{nm})$ & $4.4(1)$ & $4.5(1)$ & $4.3(1)$ & $4.4(1)$ \\
\hline Volume fraction, $\phi_{\mathrm{CSH}}$ & $0.135(17)$ & $0.196(24)$ & $0.161(20)$ & $0.202(24)$ \\
\hline Fractal exponent, $D_{\mathrm{V}}$ & $2.62(5)$ & $2.64(5)$ & $2.60(5)$ & $2.67(5)$ \\
\hline Correlation length, $\xi_{\mathrm{V}}(\mathrm{nm})$ & $63(17)$ & 47 (13) & $37(10)$ & $51(14)$ \\
\hline Packing ratio, $\eta_{\mathrm{VF}} / \eta$ & $0.37(2)$ & $0.42(2)$ & $0.41(2)$ & $0.45(2)$ \\
\hline
\end{tabular}

The numbers in parentheses are the estimated uncertainties in least significant digits.

one week and a few years. This is in agreement with previous observations $[5,14]$ that the volume fractal structure of cement paste is largely established after just one day of hydration. Clearly, significant hydration occurs after this time, as indicated by heat evolution, strength development, and other parameters. This is reflected in the SANS data as an increase in $\phi_{\mathrm{CSH}}$ and a decrease in $\xi_{\mathrm{V}}$, with a concurrent increase in the volume fractal packing ratio, $\eta_{\mathrm{VF}} / \eta$. The increase in $\eta_{\mathrm{VF}} / \eta$ can be associated with the elimination of the larger gel pores within low-density (outer product) $\mathrm{C}-\mathrm{S}-\mathrm{H}$ in regions of the paste where the hydration product layers around adjacent cement particles are growing into each other. This is discussed in more detail in Section 6.

Of particular interest here is the data for the heat-cured pastes listed in the last two columns of Table 1. In this case, while the particle size and fractal exponent are unchanged, the volume fractal regions exhibit lower correlation lengths and higher packing ratios similar to the much older paste hydrated at $20^{\circ} \mathrm{C}$. The higher packing ratio likely results from a greater mobility of the C-S-H particles at elevated temperatures, leading to a denser structure with smaller gel pores. It can also be seen from Table 1 that heat curing increases the amount of volume fractal C-S-H in the paste $\left(\phi_{\mathrm{CSH}}\right)$ but does not increase the surface area. This can be attributed to an increased loss of free surface area due to more particle-particle contacts in the more densely packed structure.

\section{Length and weight changes of cement paste during equilibrium drying}

Data relating the weight loss on drying to the drying strains can provide important information about the pore size distribution of a material. However, drying of cement paste is generally conducted under non-equilibrium conditions for which the relative humidity $(\mathrm{RH})$ inside the specimen is an unknown function of both time and position until the drying process is complete. This generates only a single data point, which is not sufficient to provide information about pore size distribution. A more useful approach is to conduct stepwise drying, taking care to reach equilibrium at each RH level. Such an experiment was first performed by Roper in the 1960s [15], and those results are now widely republished in standard concrete texts [e.g. 11] because of the insight that the general shape 
of the weight loss versus shrinkage plot provides into drying shrinkage mechanisms. Such equilibrium drying experiments can also provide information about the volume of pores emptied in different size ranges, and thus can provide information about the pore structure of the C-S-H gel in specimens cured in different ways [16,work,in,preparation]. When a porous body is dried, the pores generally empty in order of size, starting with the largest. At RH levels above $\sim 40 \%$, drying follows the wellknown Kelvin relationship between pore size and relative humidity, and the capillary stresses exerted by the liquid-vapor interfacial energy (given by the Laplace equation) generate bulk drying shrinkage. While the stress in a given pore containing a meniscus increases as the pore diameter decreases, the observed bulk shrinkage will also depend on the number of pores being emptied at a given time, that is, on the pore size distribution. Near $40 \%$ RH the rate of drying shrinkage generally levels off due to the collapse of the air-water menisci, but then increases again at lower RH as other mechanisms, such as changes in surface energy due to the removal of adsorbed water become important.

Fig. 2 (at left) shows the equilibrium drying results for an OPC paste cured for $7 \mathrm{~d}$ at room temperature. After drying to $7 \% \mathrm{RH}$ the specimen was resaturated and then dried a second time; both drying cycles are shown. The data from the second drying cycle has been shifted so that it overlays the data from the first cycle below $33 \%$ RH. It can be seen that all of the differences between first and second drying occur above 33\% $\mathrm{RH}$, corresponding to the emptying of pores greater than $\sim 2 \mathrm{~nm}$ in diameter. These differences correspond almost entirely to irreversible changes to the pore structure of the paste induced by the first drying cycle, as evidenced by the fact that there is almost no irreversible shrinkage associated with the second drying cycle. The weight loss in each humidity range is listed in
Table 2

Weight loss (\%) in different relative humidity ranges for the equilibrium drying of OPC paste (see graphs in Fig. 2)

\begin{tabular}{lllllll}
\hline Age/treatment & $100-$ & $85-$ & $76-$ & $54-$ & $33-$ & $19-7 \%$ \\
& $85 \%$ & $76 \%$ & $54 \%$ & $33 \%$ & $19 \%$ & \\
\hline 7 d (first dry) & 5.5 & 3.1 & 3.3 & 2.4 & 2.2 & 0.9 \\
7 d (second dry) & 6.8 & 1.3 & 1.8 & 2.2 & 1.8 & 1.0 \\
56 d (not plotted) & 2.8 & 2.7 & 3.7 & 4.5 & 1.9 & 1.0 \\
7 d (heat treated) & 7.6 & 1.8 & 2.7 & 3.1 & 1.9 & 0.9 \\
7 d (heat cured) & 8.5 & 2.1 & 2.3 & 3.4 & 1.8 & 0.9 \\
\hline
\end{tabular}

Table 2. It can be seen that on second drying there is more weight loss from larger (capillary) pores $(100-85 \% \mathrm{RH})$ and less weight loss from smaller pores $(85-54 \%)$ as compared to the first drying, while the weight loss from the smallest pores $(<54 \%)$ remains unchanged. Fig. 2 (at right) shows the equilibrium first drying data for OPC pastes cured under different conditions. One specimen was cured at $60{ }^{\circ} \mathrm{C}$ for $7 \mathrm{~d}$ (heat cured), and another specimen was cured for $6 \mathrm{~d}$ at room temperature and then for $1 \mathrm{~d}$ at $60{ }^{\circ} \mathrm{C}$ (heat treated). Data from these specimens are overlaid onto the data from the $7 \mathrm{~d}$ old paste cured at $20^{\circ} \mathrm{C}$. Once again it can be seen that there are significant differences in the weight loss-shrinkage relationships above $33 \%$ RH but little or no difference below 33\% RH.

The weight loss in each humidity range for the data plotted in Fig. 2, along with data from a $56 \mathrm{~d}$ old OPC paste, is listed in Table 2. Comparing the $7 \mathrm{~d}$ and $56 \mathrm{~d}$ pastes, it can be seen that extended curing at room temperature increases the volume of pores emptied between $54 \%$ and $33 \% \mathrm{RH}$. This indicates that the porosity in this range can be associated primarily with the high-density C-S-H gel, as formation of this structure is the only significant reaction that occurs after $7 \mathrm{~d}$ of hydration. Extended curing also decreases the volume of the largest pores emptied
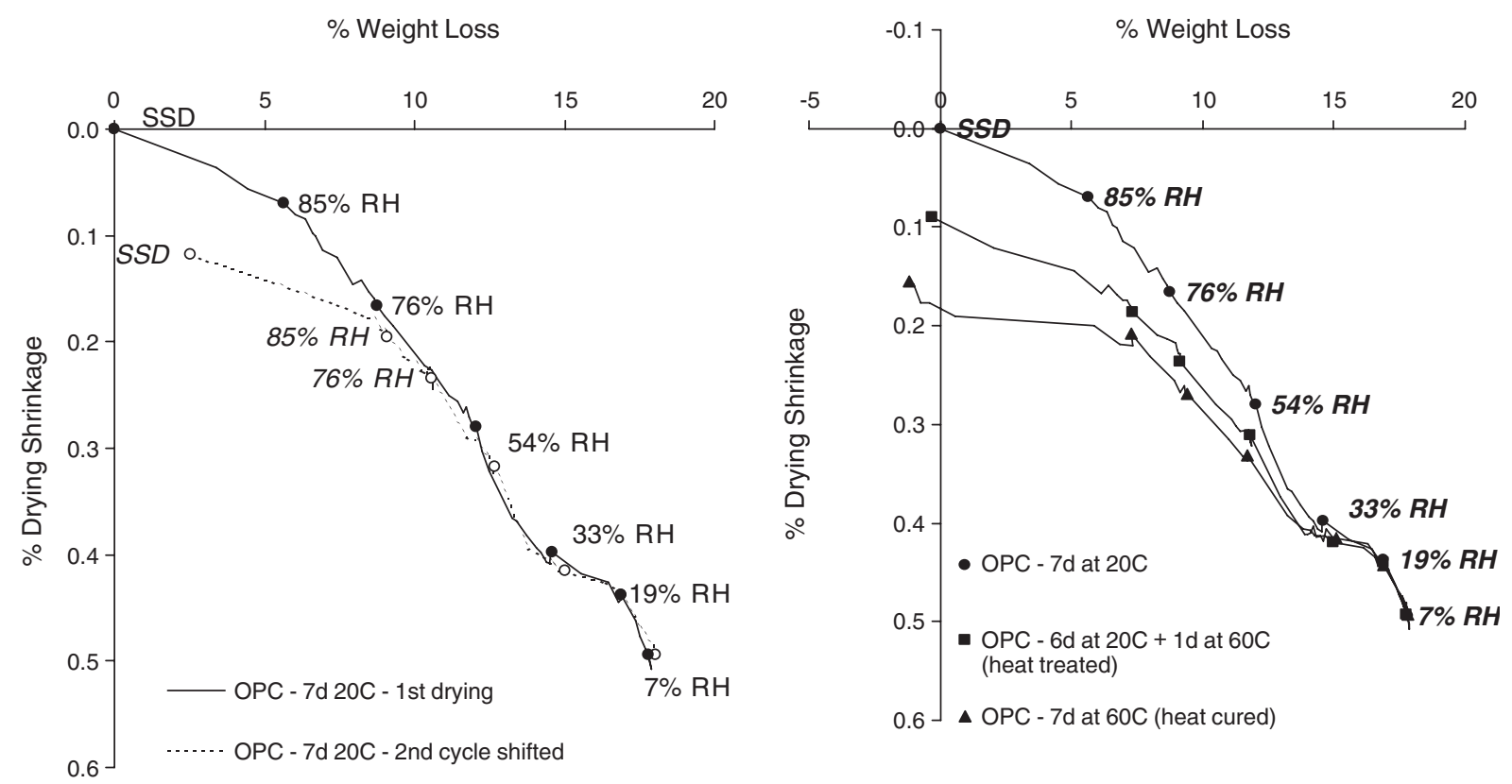

Fig. 2. Left: Equilibrium drying data for first and second drying of $7 \mathrm{~d}$ old OPC paste. Right: Equilibrium drying data for first drying of OPC paste cured under different conditions. Data is shifted to overlay the $7 \mathrm{~d}$ OPC paste (see text). 
above $85 \% \mathrm{RH}$, which is simply a result of continued hydration reducing the volume of capillary pore space in the paste.

From Fig. 2 and Table 2 it can be seen that heat curing and first drying have similar effects on the microstructure, in that the weight loss on drying to $85 \% \mathrm{RH}$ is increased, while weight loss on drying between $85 \%$ and $54 \% \mathrm{RH}$ is significantly reduced. This can be interpreted as an increase in the capillary porosity at the expense of gel porosity within the low-density C-S-H, in agreement with previous nitrogen sorption measurements of $\mathrm{C}_{3} \mathrm{~S}$ pastes cured at 25 and $60{ }^{\circ} \mathrm{C}$ [17].

\section{Nanoindentation measurements of cement paste}

The advent of instrumented indentation has given the concrete mechanics community an unprecedented opportunity for nanomechanical property exploration. The indentation technique $[18,19]$ consists of establishing contact between the indenter material (typically diamond) and the indented material whose mechanical properties are of interest. Such nanoscale instrumented indentation has, for the first time, allowed the fundamental mechanical response of small material volumes of C-S-H gel within hardened cement paste to be directly investigated and quantified $[4,20]$.

Elastic contact mechanics provides a convenient framework for linking the measured indentation modulus, $M$, with the elastic properties of the indented material [e.g. 21]. Application of this approach to cement-based materials, which are multiscale composites, poses several difficulties, as the underlying analysis relies on the self-similarity of the indentation test, which strictly holds only for homogeneous materials. However, the same principles hold for heterogeneous materials provided that the number of indentation tests is significantly increased, such that the results can be analyzed statistically. In particular, when the depth of indentation is much smaller than the size of the heterogeneity, intrinsic material properties of the individual mechanical phases can be extracted $[4,20]$. For mature cement pastes of $w / c=0.5$ it was found that an indentation depth of approximately $150 \mathrm{~nm}$ is small enough to ensure minimal interaction between separate mechanical phases, whereas an indentation depth in excess of 10-20 $\mu \mathrm{m}$ yields the homogenized (composite) properties of the bulk cement paste.

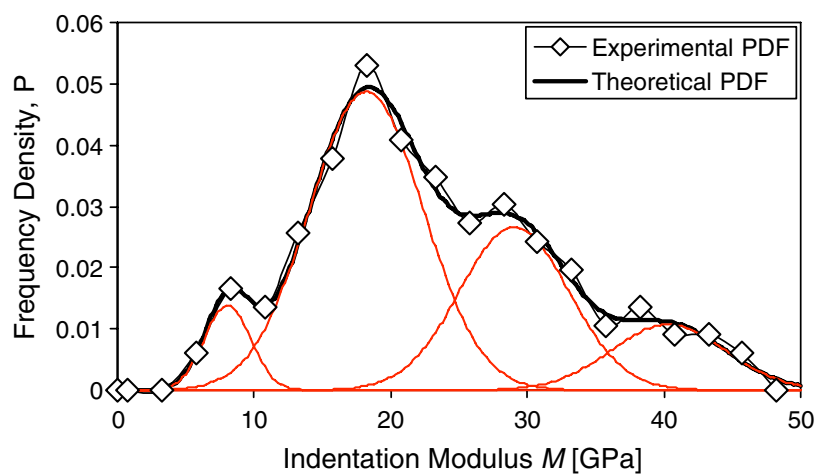

Fig. 3. Frequency plot of indentation modulus, for a WPC paste mixed at a w/c ratio of 0.5 and cured for $28 \mathrm{~d}$ at $20^{\circ} \mathrm{C}$, based on a $10 \times 10$ grid of nanoindentation measurements.
Table 3

Indentation modulus, $M$, for the LD and HD forms of C-S-H as determined from indentation measurements of WPC paste specimens mixed at a water/cement ratio of 0.5 and given the curing time and temperature indicated

\begin{tabular}{|c|c|c|c|c|}
\hline & \multirow{2}{*}{$\begin{array}{l}28 \mathrm{~d} \text { at } \\
20^{\circ} \mathrm{C}\end{array}$} & $1 \mathrm{~d}$ at $60^{\circ} \mathrm{C}$ & \multirow{2}{*}{$\begin{array}{l}28 \mathrm{~d} \text { at } \\
60{ }^{\circ} \mathrm{C}\end{array}$} & $27 \mathrm{~d}$ at $20^{\circ} \mathrm{C}$ \\
\hline & & $27 \mathrm{~d}$ at $20^{\circ} \mathrm{C}$ & & $1 \mathrm{~d}$ at $60^{\circ} \mathrm{C}$ \\
\hline & $M(\mathrm{GPa})$ & $M(\mathrm{GPa})$ & $M(\mathrm{GPa})$ & $M(\mathrm{GPa})$ \\
\hline LD C-S-H & $18.1 \pm 4.0$ & $17.8 \pm 4.3$ & $18.0 \pm 3.1$ & $18.3 \pm 3.8$ \\
\hline HD C-S-H & $31.0 \pm 4.0$ & $29.8 \pm 2.3$ & $28.5 \pm 2.6$ & $29.1 \pm 5.3$ \\
\hline
\end{tabular}

Fig. 3 shows a frequency plot of indentation modulus for a WPC paste cured at $20{ }^{\circ} \mathrm{C}$; there are two overlapping but clearly distinguishable peaks that verify the existence of two types of $\mathrm{C}-\mathrm{S}-\mathrm{H}$ in cement paste. The peaks can be deconvoluted to obtain the mean value of the indentation modulus for HD C-S-H and LD C-S-H (see Table 3). These values have been reproduced closely on several specimens with different cement compositions, w/c-ratios, and degrees of hydration $[4,20,22]$. For the present study, to test the effects of heat curing on the mechanical properties of C-S-H, nanoindentation grids were also performed on WPC paste specimens that were both heat cured and heat treated at $60{ }^{\circ} \mathrm{C}$. These results are also listed in Table 3. It can be readily observed that the characteristic values of the indentation moduli of the two types of C-S-H are essentially constant, indicating that the heat curing process does not change the intrinsic mechanical properties of the C-S-H structure. It therefore appears that these properties as measured by nanoindentation are intrinsic in nature [20].

It should be noted that what is measured by the indentation technique is the homogenized properties of the two types of C$\mathrm{S}-\mathrm{H}$ at the length scale of at least tens of nanometers, whereas it is well established that C-S-H has porosity at length scales down to about $1 \mathrm{~nm}$. Direct experimental measurement of the nanomechanical properties of solid C-S-H is currently still out of reach. However, the value of the solid C-S-H indentation modulus $\left(m_{\mathrm{s}}\right)$ can be obtained from the reverse application of a self-consistent micromechanical model, commonly employed for polycrystalline materials, to the homogenized nanoindentation data. The detailed analysis of this approach developed in [23] will be presented elsewhere. This approach yields separate estimates of the solid C-S-H indentation stiffness for the LD CS-H $\left(m_{\mathrm{s}}=65.9 \mathrm{GPa}\right)$ and for the HD C-S-H $\left(m_{\mathrm{s}}=62.9 \mathrm{GPa}\right)$. The two values are very close, suggesting that the two C-S-H structures are comprised of the same solid phase and thus that their different mechanical performance is related to differences in their nanometer-scale porosity.

More information about the porosity of the LD and HD structures can be obtained by making a forward application of the micromechanical prediction of the indentation modulus of an aggregated system of particles (here approximated by the self-consistent scheme) with an intrinsic solid stiffness of $m_{\mathrm{s}}=65 \mathrm{GPa}$ (average value of the two estimates). Fig. 4 shows the predicted indentation modulus of such a system as a function of the solid packing fraction. The contact stiffness of the solid spheres is recovered for a packing density of 1 , whereas a complete loss of mechanical performance is predicted 


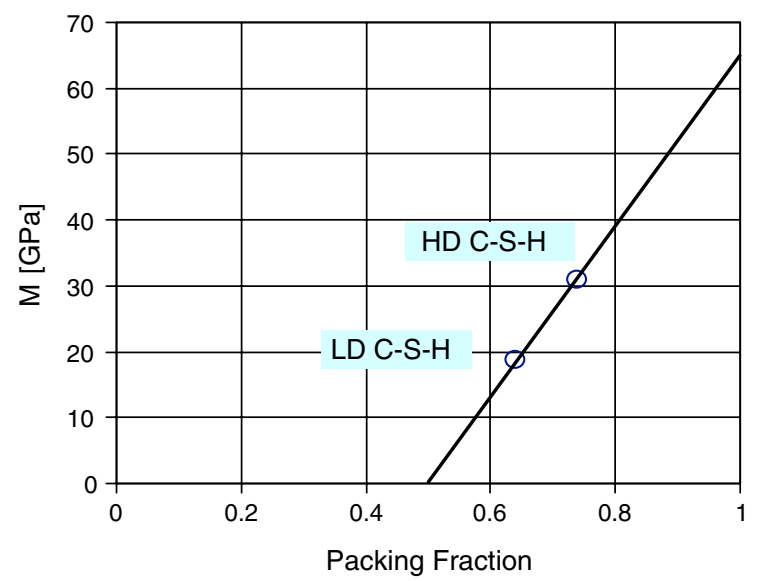

Fig. 4. Micromechanical prediction (solid line) of the indentation modulus of an aggregation of spherical particles (with $m_{\mathrm{s}}=65 \mathrm{GPa}$ ) versus the packing fraction of solid. The experimental modulus values of LD and HD C-S-H are also plotted onto the prediction line.

at a packing density of 0.5 . The latter value is close to the random loose packing of spheres, which is the lowest packing density for a mechanically stable agglomerate of spheres. The experimental average modulus values for the two types of C-S$\mathrm{H}$, also plotted in Fig. 4, correspond to packing densities of 0.64 for the LD C-S-H and 0.74 for the HD C-S-H. As discussed in the next section, these values match the porosity values of $\mathrm{LD}$ and HD C-S-H predicted by the quantitative colloid model of CS-H gel [2].

\section{Extended colloid model of C-S-H - effects of heating and drying}

The results reported here provide new boundary conditions that can be used to generate a refined model of the nanostructure of C-S-H gel. The original "colloid" model of C-S-H [2] was based on density and surface area values for C-S-H gel determined by a variety of techniques. The measured value of density or surface area was found to increase as the size of the smallest features that could be probed decreased (within the range of about $1-50 \mathrm{~nm}$ ), which is a hallmark of a fractal structure. This is in agreement with SANS measurements of cement paste, which give more direct evidence of a fractal structure. Fig. 5 shows a schematic of the development of a volume fractal structure with the general features predicted by the colloid model.

To calibrate the colloid model, the size and density of the smallest units, or particles, of C-S-H, which form the building blocks of the fractal system of solid particles and gel pores, must be assigned. A range of density values for $\mathrm{C}-\mathrm{S}-\mathrm{H}$ has been reported in the literature [24] (e.g., for D-dried C-S-H), and for any particular value within this range the model describes a selfconsistent pore structure that varies with scale. In the original version of the model, two fundamental density values were identified, but, as was stated in the original paper [2]: "the results of this model do not determine which density of the basic unit is correct, although there might be a slight bias toward the higher density."

We propose here that the smallest units that form the basis of the fractal solid/pore system are the $\sim 5 \mathrm{~nm}$ features identified originally as "globules," and not the smaller "basic units". Although the globules have an internal structure and contain a certain amount of water that is removed at low RH values, the associated spaces are too small to be considered true pores. We also propose here that a higher density assigned to a globule is the correct value. As in the original model, the globules pack together to form larger structures with lower densities, and in mature pastes there are distinct "high-density" (HD) and "lowdensity" (LD) morphologies of C-S-H gel.

Recent results from SANS (e.g. Table 1) indicate that the globule has a diameter of about $4.4 \mathrm{~nm}$ (compared to the value of $5.2 \mathrm{~nm}$ used in the original model) that does not change with drying, heat curing, or age, and a saturated density of about $2650 \mathrm{~kg} / \mathrm{m}^{3}$. This density value is slightly higher than the measured density of solid C-S-H at $11 \% \mathrm{RH}$, and is close to the average density reported for D-dried C-S-H. For an isolated globule, the specific surface area is calculated to be $515 \mathrm{~m}^{2} / \mathrm{g}$,

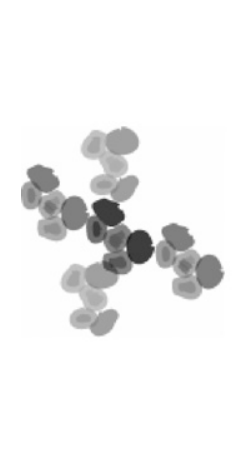

a)

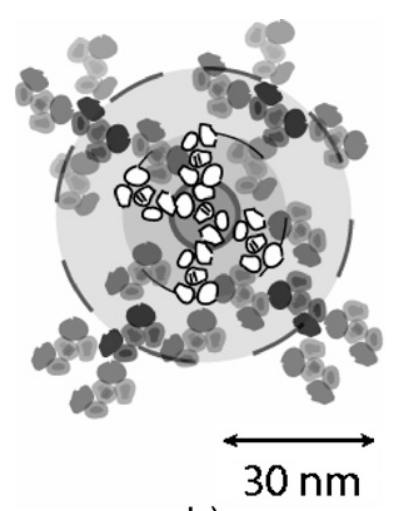

b)

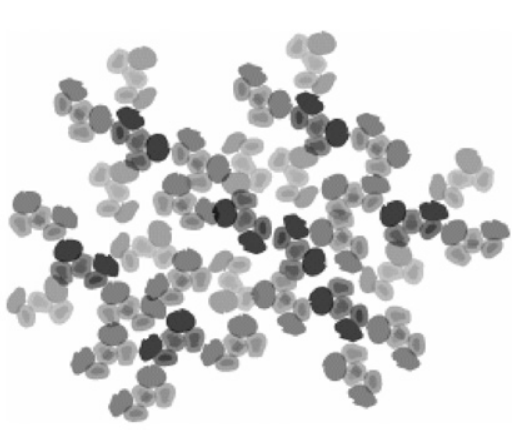

c)

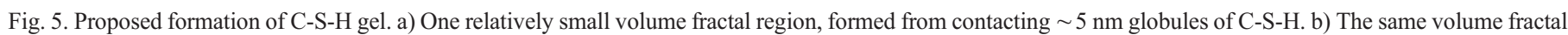

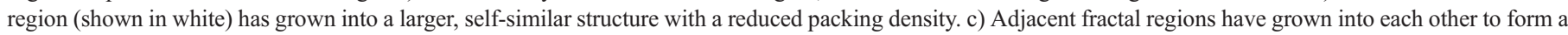

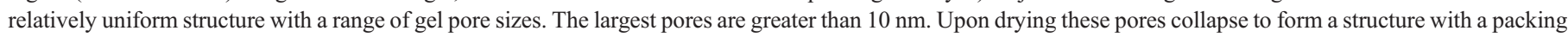
density of about 0.64 . 
which is slightly higher than the value reported in the original model. This theoretical upper limit is never approached analytically, as in all structural variations surface area is lost at particle contacts. Table 4 lists the density of C-S-H at various scales with the new calibration of the colloid model.

While the recalibration does not change the self-consistency arguments for surface area and density advanced in the original model, it does have ramifications at larger scales that are important to results reported here. A central tenet of the colloid model is that the globules pack together to form C-S-H regions with two distinct densities at larger scales. Regions of HD C-S-H contain $26 \%$ porosity (excluding, by definition, any porosity within the globule) and this remains unchanged.

In the original version of the model, the globules pack together into "LD units" approximately $20 \mathrm{~nm}$ across that contain $36 \%$ porosity (excluding the porosity within the globules), with a characteristic gel pore size slightly smaller than the particle size (i.e., a few $\mathrm{nm}$ ). At larger scales $(>100 \mathrm{~nm})$ the LD units themselves pack into a structure with a total porosity greater than $36 \%$, introducing additional pores that are, on average, somewhat larger than the particle size (i.e. $5-10 \mathrm{~nm}$ ). Intuitively, it is these larger gel pores that would be most vulnerable to collapse, and this is supported by the weight loss measurements during equilibrium drying presented in Section 4.

Fig. 6 shows the percentage of the total weight loss that occurs in various humidity ranges for different pastes, and gives pore classifications for each range. After drying and resaturation of a $7 \mathrm{~d}$ old paste, and after heat curing or heat treatment, the proportion of the porosity emptied between $85 \% \mathrm{RH}$ and $54 \%$ $\mathrm{RH}$ is significantly decreased compared to the $7 \mathrm{~d}$ control cured at room temperature, while the proportion of the porosity emptied at lower RH levels are almost unchanged. In terms of the model, this can now be interpreted as a loss of the larger gel pores between the LD units, which results in a corresponding increase in the capillary pore volume outside the C-S-H gel regions, such that the LD C-S-H at large length scales more closely represents the stable structure with $36 \%$ porosity (see Table 4). This is in good agreement with the SANS results for heat curing, particularly the higher value of the volume fractal packing ratio, $\eta_{\mathrm{VF}} / \eta$ (see Table 1), and also with SANS results for drying, which will be published separately [25]. Below 33\%

Table 4

Density and porosity of C-S-H gel at different length scales predicted by the recalibrated colloid model

\begin{tabular}{lclcl}
\hline & $\begin{array}{l}\text { Scale }^{\mathrm{a}} \\
(\mathrm{nm})\end{array}$ & $\begin{array}{l}\text { Density (pores empty) } \\
\left(\mathrm{kg} / \mathrm{m}^{3}\right)\end{array}$ & $\begin{array}{l}\text { Porosity }^{\mathrm{b}} \\
(\%)\end{array}$ & $\begin{array}{l}\text { Density }^{\mathrm{c}} \text { (pores full) } \\
\left(\mathrm{kg} / \mathrm{m}^{3}\right)\end{array}$ \\
\hline Globule & 4.4 & 2650 & 0 & 2650 \\
HD & $>15$ & 2000 & 26 & 2300 \\
LD (stable) & $>40$ & 1700 & 36 & 2050 \\
LD (early) & $>60$ & $<1700$ & $>36$ & $<2050$ \\
\hline
\end{tabular}

The stable form of LD C-S-H is obtained after heat curing or drying. The less dense "early" form has a variable structure and may persist indefinitely under ideal conditions.

${ }^{\text {a }}$ Above which structure appears uniform.

${ }^{\mathrm{b}}$ Computed from density with pores empty.

${ }^{\mathrm{c}}$ Computed from porosity.

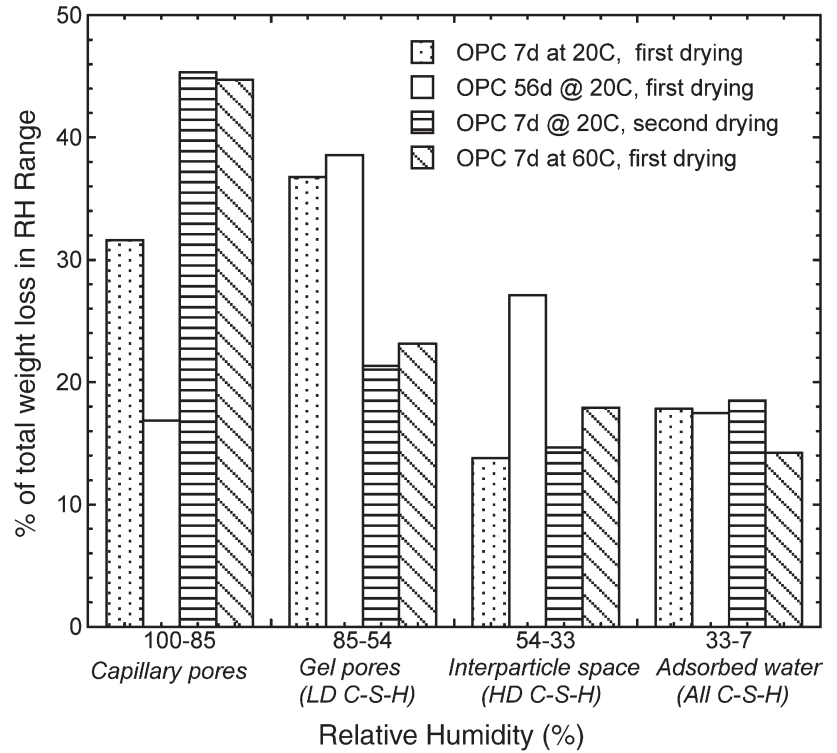

Fig. 6. Percentage of the total weight loss occurring in various RH ranges, and associated pore type.

$\mathrm{RH}$, adsorbed water and interlayer water is removed from the individual particles of both LD and HD C-S-H, a process which does not depend on the details of the gel pore structure and thus is relatively unaffected by curing conditions or previous drying. Extended curing from 7 to $56 \mathrm{~d}$ does not significantly affect the weight loss in the humidity range from $85 \%$ to $54 \%$, suggesting that the more open LD C-S-H structure that forms initially may persist under ambient saturated conditions.

As discussed in Section 5, nanoindentation measures two characteristic modulus values for $\mathrm{C}-\mathrm{S}-\mathrm{H}$ gel that are unchanged by heat curing or heat treating, and the data can be interpreted as two packing densities of the same solid phase corresponding to porosities of $36 \%$ and $26 \%$. These can be readily associated with the HD and LD C-S-H structures proposed by the colloid model, with the caveat that it is only the more stable and tightly packed LD structure that is sampled. This is supported by the fact that no change in the average modulus of the LD C-S-H structure is observed on heat curing (see Table 3).

Because the indenter tip contacts an area with dimensions on the order of $100 \mathrm{~nm}$, it must be assumed that the more loosely packed "early" version of LD C-S-H that can be present at this scale is not observed in the specimens tested to date, even those cured at room temperature. This may be explained by the fact that the size of these larger gel pores interferes with the chosen indentation depth, leading to a loss of self-similarity of the indentation test, which is at the basis of indentation analysis. In support of this proposition is the observation that the deconvoluted indentation data frequently shows a low-stiffness population (on the order of 5-8 GPa), attributable to indentation in a material dominated by large pores [23]. Another possibility is that sample preparation or drying of the sample surface prior to the nanoindentation test causes the LD C-S-H to collapse to its more stable configuration. This issue will be the subject of future investigation. 


\section{Summary}

The effects of heat curing and drying on the nanometer-scale structure of cement paste have been analyzed using small-angle neutron scattering (SANS), equilibrium drying measurements, and nanoindentation. The SANS and equilibrium drying results indicate that hydration of cement paste at $20{ }^{\circ} \mathrm{C}$ forms a lowdensity (LD) C-S-H gel structure with a range of gel pore sizes and a relatively low packing fraction of solid particles. This initial fine structure may persist indefinitely under saturated conditions, as additional C-S-H gel with a denser morphology forms. However, if the paste is dried or is cured at elevated temperatures $\left(60{ }^{\circ} \mathrm{C}\right.$ or greater) the originally formed low-density structure collapses toward a denser and more stable configuration with fewer large gel pores and a total gel porosity of $36 \%$, resulting in a greater amount of capillary porosity in the paste.

Nanoindentation measurements of pastes cured at different temperatures demonstrate the existence of two C-S-H structures with different characteristic values of the indentation modulus. The value of the modulus of the LD C-S-H is the same for all pastes tested to date, and a micromechanical analysis indicates that this value corresponds to the denser and more stable configuration of LD C-S-H. These experimental results are used to extend and recalibrate a quantitative "colloid" model of C-S-H gel, resulting in an improved understanding of the microstructural changes associated with drying and heat curing.

\section{Acknowledgements}

The Northwestern team involved in this collaborative research effort was supported by the National Science Foundation under grant CMS 0409571 and through a Graduate Student Fellowship. The SANS measurements were conducted using facilities supported in part by the National Science Foundation under Agreement No. DMR-9986442. The MIT team gratefully acknowledges the partial support of this study by Lafarge and the Reed fund at MIT. The support of MIT's Nanolab-team directed by Alan Schwartzman and Prof. Krystyn Van Vliet for the nanoindentation tests is also gratefully acknowledged.

\section{References}

[1] F.-J. Ulm, G. Constantinides, F.H. Heukamp, Is concrete a poromechanics material? - a multiscale investigation of poroelastic properties, Mater. Struct. 37 (2004) 43-58.

[2] H.M. Jennings, A model for the microstructure of calcium silicate hydrate in cement paste, Cem. Concr. Res. 30 (2000) 101-116.

[3] J.J. Thomas, J.J. Chen, A.J. Allen, H.M. Jennings, Effects of decalcification on the microstructure and surface area of cement and tricalcium silicate pastes, Cem. Concr. Res. 34 (2004) 2297-2307.
[4] G. Constantinides, F.-J. Ulm, K. Van Vliet, On the use of nanoindentation for cementitious materials, Mater. Struct. 36 (2003) 191-196.

[5] A.J. Allen, R.C. Oberthur, D. Pearson, P. Schofield, C.R. Wilding, Development of the fine porosity and gel structure of hydrating cement systems, Philos. Mag., B 56 (1987) 263-268.

[6] A.J. Allen, Time-resolved phenomena in cements, clays and porous rocks, J. Appl. Cryst. 24 (1991) 624-634.

[7] A.J. Allen, J.J. Thomas, Analysis of C-S-H gel and cement paste by smallangle neutron scattering, Cem. Concr. Res. 37 (2007) 319-324 (this issue).

[8] A.J. Allen, R.A. Livingston, Relationship between differences in silica fume additives and fine-scale microstructural evolution in cement based materials, Adv. Cem. Based Mat. 8 (1998) 118-131.

[9] K.O. Kjellsen, R.J. Detwiler, O.E. Gjorv, Backscattered electron imaging of cement pastes hydrated at different temperatures, Cem. Concr. Res. 20 (1990) 308-311.

[10] K.L. Scrivener, The effect of heat treatment on inner product C-S-H, Cem Concr. Res. 22 (1992) 1224-1226.

[11] S. Mindess, J.F. Young, D. Darwin, Concrete, 2nd ed., Prentice Hall, Upper Saddle River, NJ, 2003.

[12] A. Nonat, The structure and stoichiometry of C-S-H, Cem. Concr. Res. 34 (2004) 1521-1528.

[13] I.G. Richardson, Tobermorite/jennite- and tobermorite/calcium hydroxidebased models for the structure of C-S-H: applicability to hardened pastes of tricalcium silicate, beta-dicalcium silicate, Portland cement, and blends of Portland cement with blast-furnace slag, metakaolin, or silica fume, Cem. Concr. Res. 34 (2004) 1733-1777.

[14] J.J. Thomas, H.M. Jennings, A.J. Allen, The surface area of cement paste as measured by neutron scattering — evidence for two C-S-H morphologies, Cem. Concr. Res. 28 (1998) 897-905.

[15] H. Roper, Dimensional Change and Water Sorption Studies of Cement Paste, Symposium on Structure of Portland Cement Paste and Concrete, Washington, D.C., 1966, pp. 74-83.

[16] J.S. Gevrenov, Masters Thesis, Department of Materials Science and Engineering, Northwestern University, Evanston, IL, 2005.

[17] A. Bentur, R.L. Berger, J.H. Kung, N.B. Milestone, J.F. Young, Structural properties of calcium silicate pastes: II, effect of curing temperature, J. Am. Ceram. Soc. 62 (1979) 362-366.

[18] Y.T. Cheng, C.M. Cheng, Scaling, dimensional analysis and indentation measurements, Mater. Sci. Eng. R44 (2004) 91-149.

[19] W.C. Oliver, G.M. Pharr, Measurement of hardness and elastic modulus by instrumented indentation, J. Mater. Res. 19 (2004) 3-20.

[20] G. Constantinides, F.-J. Ulm, The effect of two types of C-S-H on the elasticity of cement-based materials: results from nanoindentation and micromechanical modeling, Cem. Concr. Res. 34 (2004) 67-80.

[21] I. Sneddon, The relation between load and penetration in the axisymmetric Boussinesq problem for a punch of arbitrary profile, Int. J. Eng. Sci. 3 (1965) 47-57.

[22] P. Acker, Micromechanical analysis of creep and shrinkage mechanisms, in: F.J. Ulm, Z.P. Bazant, F.H. Wittmann (Eds.), Creep, Shrinkage, and Durability Mechanics of Concrete and other Quasi-Brittle Materials, Elsevier Science Ltd., New York, 2001, pp. 15-25.

[23] G. Constantinides, F.-J. Ulm, Invariant mechanical properties of calciumsilica-hydrates (C-S-H) in cement-based materials: instrumented nanoindentation and microporomechanical modeling, (PhD Dissertation), MITCEE Res. Rep. R05-03, Cambridge, MA, 2005.

[24] H.F.W. Taylor, Cement Chemistry, 2nd ed., Thomas Telford, London, 1997.

[25] J.J. Thomas, A.J. Allen, H.M. Jennings, work in preparation. 